\title{
Electromagnetic Conception of Color Vision in Additive Mixing of Colors. Application in the Photography. Art and Psychology
}

\author{
Ignat Ignatov $^{1 *} \quad$ Kevork Vanlyan ${ }^{2}$ \\ 1. DSc, professor, Scientific Research Center of Medical Biophysics (SRCMB), \\ 32 N. Kopernik St., Sofia 1111, Bulgaria \\ 2. BSc., photographer, Scientific Research Center of Medical Biophysics (SRCMB) \\ 32 N. Kopernik St., Sofia 1111, Bulgaria
}

\begin{abstract}
Vision (visual perception) is a process of psycho-physiological processing of the images of surrounding objects, carried out by the visual system, which allows getting an idea of the size, shape and color of surrounding objects, their relative position and distance between them. The vision analyzer can perceives waves of a certain length from 380 to $770 \mathrm{~nm}$ with pigments - rhodopsin and iodopsin. The aim of the research is the mechanism of color perception by the visual analyzer having the ability to analyze certain ranges of the optical spectrum. The colors were studied along with an analysis of the additive mixing of two colors. In 2008 Marinov and Ignatov shown that at the mixing of electromagnetic waves with different wavelengths, the visual analyzer perceive them as separate or average wave length corresponding to mix color.
\end{abstract}

Keywords: vision, rhodopsin, iodopsin, additive color mixing

DOI: $10.7176 / \mathrm{JMPB} / 64-02$

Publication date: February $29^{\text {th }} 2020$

\section{Introduction}

Light rays from subjects pass through the optical system of the eye (cornea, lens and vitreous body) and onto the retina, where the light-sensitive photoreceptor cells (rods and cones) are located. Light incidental on the photoreceptors, triggers a cascade of biochemical reactions of visual pigments. The rhodopsin is responsible for the perception of electromagnetic radiation in the visible range and in turn, - the occurrence of nerve impulses, which are transmitted through the following retinal neurons and further to the optic nerve. The optic nerve carries the nerve impulses into the lateral geniculate body - subcortical center of vision, and thence to the cortical center, located in the occipital lobe of the brain, where the visual image is formed.

During the first decade of XXI century have been obtained new data revealing the molecular basis of visual perception. It were identified visual molecules of eucariotes (rhodopsin, iodopsin) and procariots (bacteriorhodopsin) involved in light perception and cleared up the mechanism of their action.

\section{Materials and methods}

\subsection{Absorption Spectra}

Absorption spectra of pigments were recorded on programmed DU-6 spectrophotometer ("Beckman Coulter", USA) at $280 \mathrm{~nm}$ and $750 \mathrm{~nm}$.

\subsection{Color Analyzing}

Colors were analyzed by using color analyzer "Tsvetan" ("Photopribor", Cherkassk, Ukraine). Operating relative absorbance, $\%$ from -80 to 70 . Measurement error, $\pm 5 \%$. Response time from 0.4 to $63 \mathrm{sec}$. Overall dimensions, $300 \mathrm{~mm}$.

\section{Results and discussion}

\subsection{Theoretical Aspects of Molecular Basis of Vision}

The process of perception of light has a definite localization in photoreceptor light-sensitive cells of the retina. The retina in its structure is a multilayer layer of nervous tissue that is sensitive to light, which lines the inside of the back of the eyeball. Light enters through the eye and creates a complex biochemical reaction in the photoreceptor cells of the retina. Photoreceptor cells are divided into two types that due to their characteristic form are designated as rods and cones. Rods are receptors of light of low intensity; they arranged in a colored layer of the retina, in which is synthesized photochromic protein rhodopsin, responsible for color perception. Cones on the contrary contain a group of visual pigments (iodopsin), and adapted to distinguish different colors. Rods can persive black and white images in the dim light, cones - to carry out color vision in bright light. Human retina contains approximately 3 million of cones and 100 million of rods. Their dimensions are very small - the length of about 40-50 $\mu \mathrm{m}$, the diameter from 1 to $4 \mu \mathrm{m}$.

Electrical signals generated by the rods and cones, are handled by other retinal cells. They are bipolar and ganglion cells before the electrical signals to be transmitted to the brain via the optic nerve (5). Cones are 
approximately 100 times less sensitive to light than rods, but much better perceive the rapid movement. The wand can be stimulated by a single photon. Cascade of molecular interactions enhances this "quantum" of information into a chemical signal, which is then perceived by the nervous system. The degree of enhancement signal varies depending on ambient light. The rods are more sensitive under low than inder bright light. As a result, they operate effectively in a wide range of ambient light. Sensory system of rods is packed up in clearly distinguishable cellular substructure that can be easily selected and investigated in vitro in isolated state. This property makes them as indispensable object for further structural-functional studies as well as studies of photoreceptor pigments (rhodopsin, iodopsin).

\subsection{Rhodopsin and its Structural and Functional Properties}

Rhodopsin (6) is photoreceptor proteins of rod cells, which absorbs a photon and creates a biochemical response constituting a first step in a chain of events that provide vision. Rhodopsin consists of two components - a colorless protein opsin and a chromophore component 11-cis-retinal residue (Fig. 1). The absorption of a light photon by 11-cis-retinal "turns on" the enzymatic activity of opsin and further photosensitive biochemical cascade of reactions that are responsible for vision.

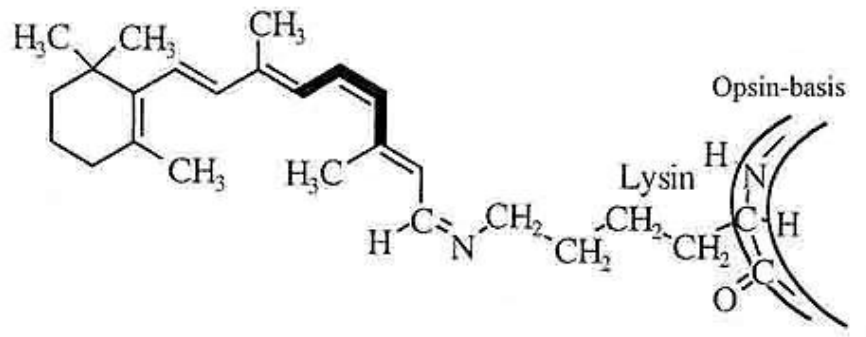

Figure 1. Configuration of photosensitive chromophore of rhodopsin in the basic (unexcited) phase (at the double bond is marked 11-cis-configuration)

\subsection{Iodopsin}

Iodopsin is a violet, light-sensitive pigment of the retinal cone cells, responsible for color vision, and close analogue of rhodopsin. This pigment consists of a protein photopsin linked with a chromophore, retinal residue. According to the three-component theory of vision, it is believed that there have to be three types of this pigment and accordingly three types of cones that are sensitive to blue, green and red light. Iodopsin consists of three pigments - hlorolab, eritrolab and tsianolab. With the densitometry method W. Rushton studied the coefficient of light absorption in the photo layers of the retina with different wavelengths. The hlorolab pigment absorbs the rays corresponding to yellow-green (450-630 nm absorption band), the eritrolab - yellow and red (500-700 $\mathrm{nm})$, and the tsianolab - blue-green $(500-700 \mathrm{~nm})$ parts of the visible spectrum.

\subsection{The Mechanism of Color Vision}

The retina has three types of cone cells $-\mathrm{S}, \mathrm{M}$ and L cells, having a different sensitivity to different parts of the visible range of the spectrum (Fig. 2). The cone cells of S type have a spectral range from 400 to $500 \mathrm{~nm}$ (maximum at 420-440 nm), M type - from 450 to $630 \mathrm{~nm}$ (maximum at 534-555 nm), L type - from $500 \mathrm{~nm}$ to $700 \mathrm{~nm}$ (maximum at $564-580 \mathrm{~nm}$ ). As the curves of the sensitivity of the cone cells overlap, it is impossible for monochromatic light to stimulate only one type of cone cells.

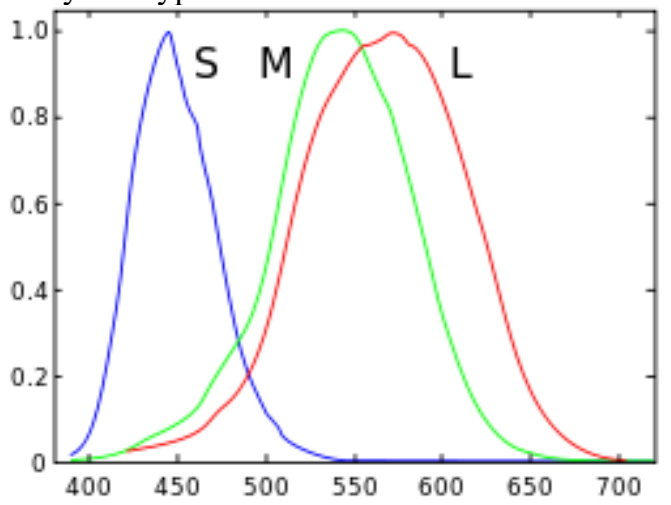

Figure 2. Spectral sensitivity of the different types of receptor cells (cones) in the retina

The other types of cone cells react though to a lesser degree. The set of all possible values of the color 
combinations causing a visual reaction determines the human color space. Human brain generally can discern approximately 10 million of different colors. The electromagnetic waves spectrum stimulates the different types of cone cells from the three types S, L and M to a different degree. The red light stimulates the L cone cells more than the $\mathrm{M}$ cone cells. The blue light stimulates the $\mathrm{S}$ cone cells in the strongest way. The yellow-green light provides a strong stimulation to the $\mathrm{L}$ and $\mathrm{M}$ cone cells, and a weaker stimulation to the $\mathrm{S}$ cone cells. The brain then combines the information from all types of cone cells for different wavelengths and analyzes them as different colors.

\subsection{Studying of Additive Mixing of Colors}

The analyses for the activity of the three types of cones $-\mathrm{S}, \mathrm{L}$ and $\mathrm{M}$ in the perception of colors also shows how the brain "deciphers" the colors. The foundation of this analysis, shown in figure 3, was made by M. Marinov and I. Ignatov in 2008. However, it is not clear whether the green color we perceive is a combined effect of yellow and blue, or whether it corresponds to a wavelength of the green color from the visible spectrum. Our brain can register the colors, i.e. the green color as a spectrometer, with certain lengths of the electromagnetic waves. It can also register the green color as a mixture of yellow and blue. The full perception of colors by the visual analyzer cannot be defined by a spectrometer.

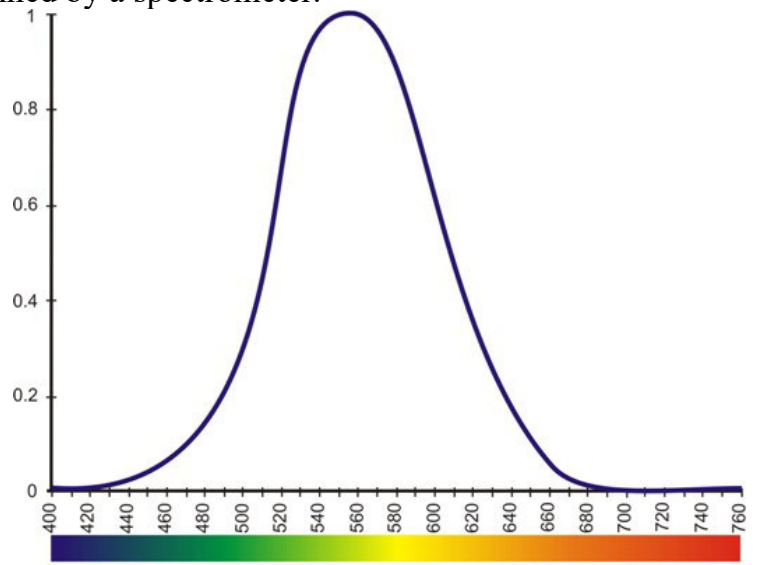

Figure 3. Spectral sensitivity of the visual analyzer

As an example in the mixing of electromagnetic waves that correspond to green and red color, yellow color is obtained. In the mixing of green and red, no medium color is obtained; the brain therefore perceives it as yellow color. When there is an emission of electromagnetic waves that correspond to green and red color, the brain adopts an "average decision" - yellow (Fig. 4). Analogously, for the yellow and blue color, the brain adopts an "average decision" - green. This means that a spectral mixing of colors is observed between the blueyellow and green-red pairs. In its turn, green and blue color is perceived as cyan. Vision sensitivity is at its lowest for the violet, blue and red color. The mixing of electromagnetic waves that correspond to blue and red color is perceived as violet. In the mixing of electromagnetic waves that correspond to more colors, the brain does not perceive them as separate or average, but as a white color. Thus the notion of color is not determined solely by the wavelength (Marinov, Ignatov, 2008; Ignatov, Moain, 2013).

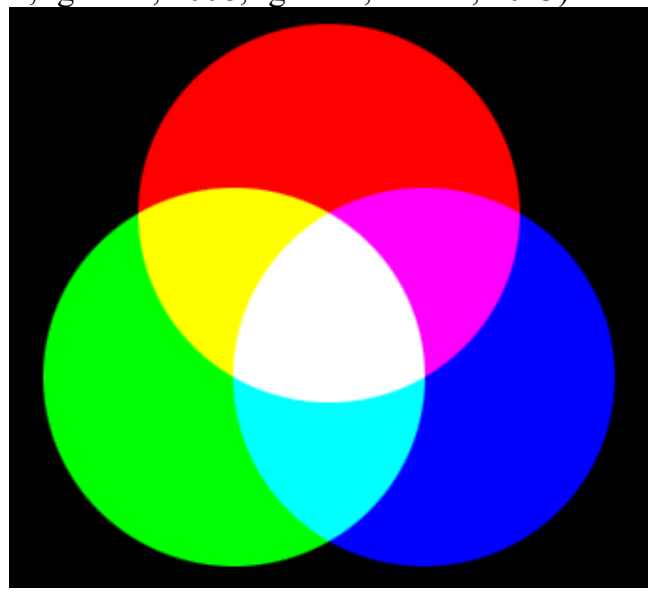

Figure 4. Additive mixing of colors 


\subsection{Electromagnetic conception in art and psychology}

In his dissertation in Glasgow University Linder shows the electromagnetic conception for the eyesight (Marinov, Ignatov, 2008; Ignatov, Moain, 2013) as scientific explanation for observation of colors.

The violet color of snow on the picture of Alexander Ignatov is with additive mixing of blue color of sky and red of sunset (Fig. 5a). For the dress, which original is with blue and black color from the figure 5 there is observation of additive mixing of colors. The people, who see the dress with blue and black color from the figure (Fig. 5b) they have bigger ability to observe the blue color as mix of cyan and violet. These people, who see the blue as monochromatic color (a) and more difficult are mixing the colors can see the dress with "non-real" white and golden color (Ignatov, 2015).
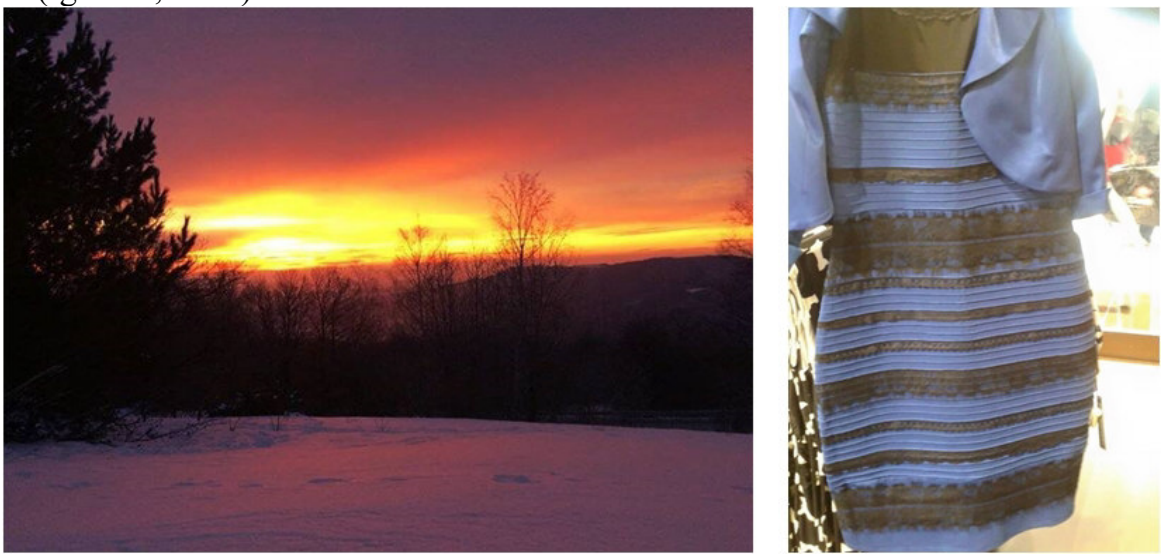

Figure 5. Sunset, Vitosha, Photo: Alexander Ignatov (a) / Dress with additive mixing of colors (b)

The analysis is performed by brain, and the notion of color is at its essence a product of our consciousness (Marinov, Ignatov, 2019).

The Russian scientists Fazlyyyakhmatov and Antipov are connected the electroamgnetic conception for vision with laws of art..

A person who is calmer, more balanced and with good psychological status the additive mixing colors is with less contrast. His perceptions are more pronounced (Ignatov, 2019).

\section{Conclusions and discussion}

The mechanism of color perception by the visual analyzer have been carried out by the author the properties of iodopsin and rhodopsin.

A further research into the function of rhodopsin and other retina affiliated chromo-proteins as iodopsin will allow investigate in detail the mechanism of visual perception of light for better treatment of functional eye diseases in ophthalmology.

The electromagnetic vision concept, created in 2008 by Marinov and Ignatov, makes it possible to make analyzes of the functional state of the visual analyzer with additive color mixing. Applications in psychology and art are shown.

\section{Acknowledgements}

The author wishes to thank Vitaly Shvetz, Dmitry Skladnev and Parashkeva Tzaneva for their cooperation in the research. Also author would likes to commemorate the memory of Prof. Marin Marinov (1928-2009) and Oleg Mosin (1966-2016).

\section{References}

Fazlyyyakhmatov, V.\&Antipov, M. (2019) Creative and Cognitive Perception of the Planar Images, Int J Edu Sci, 27 (1-3): 70-77.

Hauff, A. (2019) The Know It All Guide To Color Psychology In Marketing + The Best Hex Chart, CoSchedule Blog

Hubel, D. (1995) Eye, Brain and Vision. Scientific American Library Series (Book 22), 2nd edition, New York: W.H. Freeman Publ., 1-256.

Hunt, R.W.G. (2004). The Reproduction of Colour, (6th ed.) Chichester: Wiley-IS\&T Series in Imaging Science and Technology, 724 .

Ignatov, I.\&Mosin, O.V. (2013) Process of Perception of Light and Evolution of Sight at the Higher Animals and Humans. Naukovedenie, 3, 1-19 [in Russian]

Ignatov, I. \&Mosin, O.V. (2014) Photoreceptors in Visual Perception and Additive Color Mixing. Bacteriorhodopsin in Nano- and Biotechnologies, Advances in Physics Theories and Applications, 27: 20 - 
37.

Ignatov, I.\&Mosin, O. V. (2014) Visual Perception and Electromagnetic Conception for the Eyesight. Rhodopsin and Bacteriorhodopsin in Nano- and Biotechnologies, Journal of Health, Medicine and Nursing, 4: 1-20.

Ignatov, I..\&Mosin,O.V. (2015) Physiology of Rhodopsin and Iodopsin. Electromagnetic Conception for the Eyesight in Additive Colors, Journal of Health, Medicine and Nursing, 9:15-33.

Ignatov, I.\&Mosin, O.V. (2015) Electromagnetic Conception for the Eyesight in Additive Mixing of Colors, Journal of Health, Medicine and Nursing, 10, 65-83.

Ignatov, I. \&Mosin, O.V. (2015) Electromagnetic Conception for the Eyesight. Explanation for what the People See Different Colors in Additive Mixing, Journal of Medicine, Physiology and Biophysics, 11, 39-57.

Ignatov, I.\&Mosin,O.V. (2014) Studying of Phototransformans of Light Signal by Photoreceptor Pigments Rhodopsin, Iodopsin and Bacteriorhopsin and Additive Mixing of Colors, Journal of Medicine, Physiology and Biophysics, 3: 30-47.

Ignatov, I.\&Mosin,O.V. (2014) Visual Perception. Electromagnetic Conception for the Eyesight. Rhodopsin and Bacteriodopsin, Journal of Medicine, Physiology and Biophysics, 2:1-19.

Liang, Y., Fotiadis, D., Maeda, T. et al. (2004) Rhodopsin Signaling and Organization in Heterozygote Rhodopsin Knockout Mice, J. Biol. Chem., 279, 48189-48196.

Lipkin, V.M. (2001) Visual system. mechanisms of transmission and amplification of the visual signal in eye retina. Soros Educational Journal, 7(9), 2-8 [in Russian].

Lander, H. (2015) The Presence of Illusion, Magic and Virtual Reality, Glasgow School of Art, Glasgow University, Dissertation for Master in Fine Art.

Marinov, M.\& Ignatov, I. (2008) Color (Kirlian) Spectral Analysis. Color Observation with Visual Analyzer, Euromedica, 32.

Neugebauer, D.Ch., Zingsheim, H.P., \& Oesterhelt, D. (1978) Recrystallization of the Purple Membrane in vivo and in vitro, Journal Molecular Biology, 123, 247-257.

Nathans, J., Thomas, D.\& Hogness, D.S. (1986) Molecular Genetics of Human Color Vision: The Genes Encoding Blue, Green, and Red Pigments. Science, 232 (47), 193-202.

Hargrave, P.A., McDowell, J.H., Curtis, D.R. et al. (1983) The structure of bovine rhodopsin. Biophys. Struct. Mech., 9, 235-244.

Rushton, W.A.H. (1958). In: Visual Problems of Colour, N.P.L. Sump. (Ed.) London: Her Majesty's Stationary Office, 1, 71-101.

Schertler, G.F., \& Hargrave, P.A. (1995) Projection Structure of Frog Rhodopsin in Two Crystal Forms, Proc. Natl. Acad. Sci. U.S.A., 92, 11578-11582.

Wyszecki, G., \& Stiles, W.S. (1982) Color Science: Concepts and Methods, Quantitative Data and Formulae, (2nd ed.), New York: Wiley-IS\&T Series in Pure and Applied Optics, 935. 